\title{
ON THE GROWTH RATE OF SOLUTIONS TO GROSS-NEVEU AND THIRRING EQUATIONS
}

\author{
HyungJin HuH
}

\begin{abstract}
We study the growth rate of $H^{1}$ Sobolev norm of the solutions to Gross-Neveu and Thirring equations. A well-known result is the double exponential rate. We show that the $H^{1}$ Sobolev norm grows at most an exponential rate $\exp \left(c t^{2}\right)$.
\end{abstract}

\section{Introduction}

We study the following Cauchy problem of the nonlinear Dirac equations

$$
\begin{aligned}
& i\left(\partial_{t} u+\partial_{x} u\right)+m v=\partial_{\bar{u}} W(u, v), \\
& i\left(\partial_{t} v-\partial_{x} v\right)+m u=\partial_{\bar{v}} W(u, v), \\
& u(x, 0)=u_{0}(x), \quad v(x, 0)=v_{0}(x),
\end{aligned}
$$

where $u, v: \mathbb{R}^{1+1} \rightarrow \mathbb{C}$ and $m(\geq 0)$ is a mass. The potential $W$ takes the form

$$
W=a_{1}|u|^{2}|v|^{2}+a_{2}(\bar{u} v+\bar{v} u)^{2},
$$

where $a_{1}, a_{2}$ are real constants and $\bar{u}$ is a complex conjugate of $u$.

The system (1.1) with $W=4|u|^{2}|v|^{2}$ is called Thirring model and an initial value problem of it has been studied by several authors $[3,5,8,12]$. It is well known in [5] that the problem is globally well posed in Sobolev space $H^{1}(\mathbb{R})$. Low regularity well-posedness was discussed in $[3,8,12]$ showing that there exists a time $T>0$ and solution $u, v \in C\left([0, T], H^{s}(\mathbb{R})\right)(s \geq 0)$. Especially global existence from $L^{2}$ initial data has recently been proved in [3].

The system (1.1) with $W=\frac{1}{4}(\bar{u} v+\bar{v} u)^{2}$ is called Gross-Neveu model [7] and an initial value problem of it has been studied in $[9,13]$. The global existence of the solution in $H^{1}$ was proved in [9] where an $L^{\infty}$ bound of the solution is obtained by applying local form of charge conservation.

The spectral stability of solitary wave solutions to nonlinear Dirac equations has been studied in $[1,2,4]$. They show that the solitary waves are spectrally stable through analysis of the spectrum of linearization at a solitary wave. The orbital stability of solitary wave of Thirring model is proved recently in [11].

Received December 11, 2013.

2010 Mathematics Subject Classification. 35L45, 35Q41, 35F25, 81Q05.

Key words and phrases. Gross-Neveu, Thirring, Sobolev norm, $L^{\infty}$ bound. 
Here we are interested in the growth rate of the $H^{1}$ norm. Note that the system (1.1) has the charge conservation

$$
\int_{\mathbb{R}}|u(x, t)|^{2}+|v(x, t)|^{2} d x=\int_{\mathbb{R}}\left|u_{0}(x)\right|^{2}+\left|v_{0}(x)\right|^{2} d x .
$$

A well-known result in $[5,9]$ is that the solution of (1.1) grows at most in a double exponential, i.e.,

$$
\|u(t)\|_{H^{1}(\mathbb{R})} \leq c_{1} \exp \left(\exp \left(c_{2} t\right)\right),
$$

where $c_{1}$ and $c_{2}$ are constants. For the massless $(m=0)$ Thirring model in [8], we can derive an uniform bound $\|u(t)\|_{H^{1}} \leq c\|u(0)\|_{H^{1}}$ by using explicit solution representation. A global bound on the $H^{1}$ norm of the small $L^{2}$ solutions to the massive $(m>0)$ Thirring equation is obtained in [11] by deriving a new conserved quantity. The following is our main result.

Theorem 1.1. Consider the initial value problem (1.1) with $u_{0}, v_{0} \in H^{1}(\mathbb{R})$. Then we have the following upper bound

$$
\|u(t)\|_{H^{1}(\mathbb{R})}+\|v(t)\|_{H^{1}(\mathbb{R})} \leq c_{1} \exp \left(c_{2} t^{2}\right) .
$$

To prove Theorem 1.1, we will estimate $\|u(t)\|_{L^{\infty}}$. For the massive Thirring $[5,6,10]$ and Gross-Neveu [9] equations, $L^{\infty}$ norm of solutions was controlled by $L^{\infty}$ norm of initial data in the following way

$$
\|u(t)\|_{L^{\infty}} \leq c_{1} e^{c_{2} t} .
$$

Here we improve the above bound by $\|u(t)\|_{L^{\infty}} \leq c_{1}+c_{2} t^{\frac{1}{2}}$. Then Theorem 1.1 is proved in Section 2.

\section{Proof of Theorem 1.1}

To begin with, let us recall basic known facts. Global existence of the solution to $(1.1)$ in Sobolev space $H^{1}(\mathbb{R})$ was proved in $[5,9]$.

Theorem 2.1. For initial data $u_{0}, v_{0} \in H^{1}(\mathbb{R})$, there exists a global solution $(u, v)$ of $(1.1)$ satisfying

$$
u, v \in C\left([0, \infty), H^{1}(\mathbb{R})\right),
$$

where $u, v$ depend continuously on the initial data.

For a simple presentation of proof of Theorem 1.1, we only consider the massive Gross-Neveu equation. For the case of the massive Thirring model, the proof is similar and easier.

For the potential $W=\frac{1}{4}(\bar{u} v+\bar{v} u)^{2}$, the equation (1.1) takes the form

$$
\begin{aligned}
i\left(\partial_{t} u+\partial_{x} u\right)+m v & =\operatorname{Re}(\bar{u} v) v, \\
i\left(\partial_{t} v-\partial_{x} v\right)+m u & =\operatorname{Re}(\bar{u} v) u .
\end{aligned}
$$


To estimate $\left\|\partial_{x} u(t)\right\|_{L^{2}}$ and $\left\|\partial_{x} v(t)\right\|_{L^{2}}$, we take derivative $\partial_{x}$ on (2.1) and obtain

$$
\begin{aligned}
& \partial_{t}\left|u_{x}\right|^{2}+\partial_{x}\left|u_{x}\right|^{2}+2 m \operatorname{Im}\left(v_{x} \bar{u}_{x}\right)=2 \operatorname{Re}(\bar{u} v) \operatorname{Im}\left(v_{x} \bar{u}_{x}\right)+2 \partial_{x}(\operatorname{Re}(\bar{u} v)) \operatorname{Im}\left(v \bar{u}_{x}\right), \\
& \partial_{t}\left|v_{x}\right|^{2}-\partial_{x}\left|v_{x}\right|^{2}+2 m \operatorname{Im}\left(u_{x} \bar{v}_{x}\right)=2 \operatorname{Re}(\bar{u} v) \operatorname{Im}\left(u_{x} \bar{v}_{x}\right)+2 \partial_{x}(\operatorname{Re}(\bar{u} v)) \operatorname{Im}\left(u \bar{v}_{x}\right),
\end{aligned}
$$

which leads to

$$
\begin{aligned}
& \partial_{t}\left(\left|u_{x}\right|^{2}+\left|v_{x}\right|^{2}\right)+\partial_{x}\left(\left|u_{x}\right|^{2}-\left|v_{x}\right|^{2}\right) \\
= & 2 \partial_{x}(\operatorname{Re}(\bar{u} v)) \operatorname{Im}\left(v \bar{u}_{x}\right)+2 \partial_{x}(\operatorname{Re}(\bar{u} v)) \operatorname{Im}\left(u \bar{v}_{x}\right) \\
\leq & 2\left(|v|^{2}\left|u_{x}\right|^{2}+2|u||v|\left|u_{x}\right|\left|v_{x}\right|+|u|^{2}\left|v_{x}\right|^{2}\right) .
\end{aligned}
$$

Integrating on $\mathbb{R}$, we obtain

$$
\begin{aligned}
\frac{d}{d t} \int_{\mathbb{R}}\left(\left|u_{x}\right|^{2}+\left|v_{x}\right|^{2}\right)(x, t) d x & \leq 4 \int_{\mathbb{R}}\left(|v|^{2}\left|u_{x}\right|^{2}+|u|^{2}\left|v_{x}\right|^{2}\right)(x, t) d x \\
& \leq 4\left(\|u\|_{L^{\infty}}^{2}+\|v\|_{L^{\infty}}^{2}\right) \int_{\mathbb{R}}\left|u_{x}\right|^{2}+\left|v_{x}\right|^{2} d x .
\end{aligned}
$$

Then Gronwall's inequality gives a bound

$$
\begin{aligned}
& \left\|\partial_{x} u(t)\right\|_{L^{2}}^{2}+\left\|\partial_{x} v(t)\right\|_{L^{2}}^{2} \\
\leq & \exp \left(4 \int_{0}^{t}\|u(s)\|_{L^{\infty}}^{2}+\|v(s)\|_{L^{\infty}}^{2} d s\right)\left(\left\|\partial_{x} u_{0}\right\|_{L^{2}}^{2}+\left\|\partial_{x} v_{0}\right\|_{L^{2}}^{2}\right) .
\end{aligned}
$$

To complete the proof of Theorem 1.1, we will estimate $L^{\infty}$ bound of the solution in (2.2) by applying an idea in [9]. Multiplying (2.1) by $\bar{u}$ and $\bar{v}$ respectively, we have

$$
\begin{aligned}
\partial_{t}|u|^{2}+\partial_{x}|u|^{2}+2 m \operatorname{Im}(\bar{u} v) & =2 \operatorname{Re}(\bar{u} v) \operatorname{Im}(\bar{u} v), \\
\partial_{t}|v|^{2}-\partial_{x}|v|^{2}+2 m \operatorname{Im}(\bar{v} u) & =2 \operatorname{Re}(\bar{u} v) \operatorname{Im}(u \bar{v}),
\end{aligned}
$$

which implies

$$
\partial_{t}\left(|u|^{2}+|v|^{2}\right)+\partial_{x}\left(|u|^{2}-|v|^{2}\right)=0 .
$$

Integrating (2.5) on the domain

$$
D\left(x_{0}, t_{0}\right)=\left\{(x, t) \mid 0<t<t_{0}, x_{0}-t_{0}+t<x<x_{0}+t_{0}-t\right\},
$$

we have by applying Green's Theorem

$$
\begin{aligned}
& 2 \int_{0}^{t_{0}}|u|^{2}\left(x_{0}+t_{0}-s, s\right) d s+2 \int_{0}^{t_{0}}|v|^{2}\left(x_{0}-t_{0}+s, s\right) d s \\
= & \int_{x_{0}-t_{0}}^{x_{0}+t_{0}}\left(\left|u_{0}(s)\right|^{2}+\left|v_{0}(s)\right|^{2}\right) d s \leq M,
\end{aligned}
$$

where we denote $M=\int_{\mathbb{R}}\left(\left|u_{0}(y)\right|^{2}+\left|v_{0}(y)\right|^{2}\right) d y$. Integrating (2.3) along characteristic, we have

$$
\frac{d}{d t}|u(x+t, t)|^{2} \leq 2|v(x+t, t)|^{2}|u(x+t, t)|^{2}+2 m|u(x+t, t)||v(x+t, t)|
$$


which implies

$$
\frac{d}{d t}|u(x+t, t)| \leq|v(x+t, t)|^{2}|u(x+t, t)|+m|v(x+t, t)| .
$$

Then we have

$$
\frac{d}{d t}\left(e^{-\int_{0}^{t}|v(x+s, s)|^{2} d s}|u(x+t, t)|\right) \leq m e^{-\int_{0}^{t}|v(x+s, s)|^{2} d s}|v(x+t, t)| .
$$

Integrating both sides and considering (2.6), we obtain

$$
\begin{aligned}
|u(x+t, t)| & \leq e^{\int_{0}^{t}|v(x+s, s)|^{2} d s}\left(|u(x, 0)|+\int_{0}^{t} m|v(x+s, s)| d s\right) \\
& \leq e^{M / 2}\left(|u(x, 0)|+m\left(\int_{0}^{t}|v(x+s, s)|^{2} d s\right)^{\frac{1}{2}} t^{\frac{1}{2}}\right) \\
& \leq e^{M / 2}\left(|u(x, 0)|+m(M / 2)^{\frac{1}{2}} t^{\frac{1}{2}}\right) .
\end{aligned}
$$

The similar argument applied to (2.4) leads us to

$$
|v(x-t, t)| \leq e^{M / 2}\left(|v(x, 0)|+m(M / 2)^{\frac{1}{2}} t^{\frac{1}{2}}\right) .
$$

Then we have, from (2.7) and (2.8),

(2.9) $\|u(t)\|_{L^{\infty}}^{2} \leq e^{M}\left(2\left\|u_{0}\right\|_{L^{\infty}}^{2}+m^{2} M t\right),\|v(t)\|_{L^{\infty}}^{2} \leq e^{M}\left(2\left\|v_{0}\right\|_{L^{\infty}}^{2}+m^{2} M t\right)$.

Plugging (2.9) into (2.2), we have

$$
\begin{aligned}
& \left\|\partial_{x} u(t)\right\|_{L^{2}}^{2}+\left\|\partial_{x} v(t)\right\|_{L^{2}}^{2} \\
\leq & \exp \left(8 e^{M} \int_{0}^{t} m^{2} M s+\left\|u_{0}\right\|_{L^{\infty}}^{2}+\left\|v_{0}\right\|_{L^{\infty}}^{2} d s\right)\left(\left\|\partial_{x} u_{0}\right\|_{L^{2}}^{2}+\left\|\partial_{x} v_{0}\right\|_{L^{2}}^{2}\right),
\end{aligned}
$$

which proves Theorem 1.1.

Acknowledgement. This work was supported by the National Research Foundation of Korea Grant funded by the Korean Government (NRF-2010330-B00128).

\section{References}

[1] G. Berkolaiko and A. Comech, On spectral stability of solitary waves of nonlinear Dirac equation in 1D, Math. Model. Nat. Phenom. 7 (2012), no. 2, 13-31.

[2] N. Boussaid and A. Comech, On spectral stability of the nonlinear Dirac equation, arXiv:1211.3336.

[3] T. Candy, Global existence for an $L^{2}$ critical nonlinear Dirac equation in one dimension, Adv. Differential Equations 16 (2011), no. 7-8, 643-666.

[4] A. Comech, M. Guan, and S. Gustafson, On linear instability of solitary waves for the nonlinear Dirac equation, arXiv:1209.1146.

[5] V. Delgado, Global solutions of the Cauchy problem for the (classical) coupled MaxwellDirac and other nonlinear Dirac equations in one space dimension, Proc. Amer. Math. Soc. 69 (1978), no. 2, 289-296.

[6] R. H. Goodman, M. I. Weinstein, and P. J. Holmes, Nonlinear propagation of light in one-dimensional periodic structures, J. Nonlinear Sci. 11 (2001), no. 2, 123-168. 
[7] D. J. Gross and A. Neveu, Dynamical symmetry breaking in asymptotically free field theories, Phys. Rev. D 10 (1974), 3235-3253.

[8] H. Huh, Global strong solution to the Thirring model in critical space, J. Math. Anal. Appl. 381 (2011), no. 2, 513-520.

[9] _ Global solutions to Gross-Neveu equations, Lett. Math. Phys. 103 (2013), no. 8, 927-931.

[10] D. E. Pelinovsky, Survey on global existence in the nonlinear Dirac equations in one spatial dimension, Harmonic analysis and nonlinear partial differential equations, 3750, RIMS Kôkyûroku Bessatsu, B26, Kyoto, 2011.

[11] D. E. Pelinovsky and Y. Shimabukuro, Orbital stability of Dirac solitons, Lett. Math. Phys. 104 (2014), no. 1, 21-41.

[12] S. Selberg and A. Tesfahun, Low regularity well-posedness for some nonlinear Dirac equations in one space dimension, Differential Integral Equations 23 (2010), no. 3-4, 265-278.

[13] Y. Zhang, Global solution to a cubic nonlinear Dirac equation in $1+1$ dimensions, arXiv:1304.1989.

Department of Mathematics

Chung-Ang University

SEOUl 156-756, Korea

E-mail address: huh@cau.ac.kr 